\title{
Basic Theory Research on Pragmatic Failure for Intercultural Communication
}

\author{
Jie Chen \\ College of Foreign Languages, Bohai University, Jinzhou, 121013, China \\ carnation2009@163.com
}

Keywords: intercultural communication; pragmatic failure; basic theory; second language acquisition theory; learning cognitive theory

\begin{abstract}
Pragmatic failure is an important factor affecting the smooth conduct of cross-cultural communication. In view of the difficulty of the research, this paper studies the related basic theory. First, second language acquisition theory, through structural diagram shows that theoretical principles and sets out the main points; then, study the learning cognitive theory, explained by the theory of error analysis, input theory, interlanguage theory, constructivist theory, information processing theory and connecting theory, and other theoretical framework; Finally, intercultural communication theory, explain the relationship of the theoretical framework of the three-dimensional model between the each constituent elements and role of the intercultural communicative competence cultivation. The research results of this paper have very important significance for the study of pragmatic failure in intercultural communication and English teaching.
\end{abstract}

\section{Introduction}

Pragmatic failure in intercultural communication is caused by cultural differences. Pragmatic failure will be reflected in many ways. Specifically speaking, the speaker does not see the object, regardless of the parties and social situations, status, identity or is in the process of unconsciously violating the social norms or social norms, irregularities time and space and other contrary to the target language unique cultural values, thus interruption or failure leading to communicative acts, so that the process of language communication in the face of obstacles, leading to communication cannot reach the expected results or the effect of perfect communication, error of this nature is called pragmatic failures. Focus only on the language itself, while ignoring the different language and cultural differences are the main cause of pragmatic failure [1,2]. Therefore, in intercultural communication to successfully eliminate the obstacles caused by the pragmatic failure, must understand the purpose of language and culture, to adjust their thinking set, so pragmatic empathy. With the rapid development of economic globalization, exchanges between China and other countries have become increasingly frequent, if you do not understand the communication and cultural backgrounds, ways of thinking, thinking mode, value orientation and other differences, will result in a large number of pragmatic failure, so that the communication between the two sides appear awkward, misunderstanding and other scenes, is bound to affect the communication. Pragmatic failure as one of the important factors affecting the smooth conduct of intercultural communication, pragmatic failure has attracted more and more attention of linguists. Basic theory research is the preliminary work of the research. This paper studies the related basic theory, for in-depth research to provide protection and support, to lay the foundation for the smooth conduct research work in related fields.

\section{Second Language Acquisition Theory}

Second language acquisition, or SLA, is the processes by which people learn languages in addition to their native tongue(s). Just as second language is the standard term for any language whose acquisition starts after early childhood, including what is chronologically the third or subsequent language, SLA is the acquisition of any second language [3]. The language to be learned is often referred to as the "target language" or "L2" (and SLA is often called L2A, for "L2 acquisition"). In 
the new century, the scope of the study of second language acquisition is more extensive, involving linguistics, psychology, psycholinguistics, pragmatics, social linguistics and many respects. Second language acquisition theory is shown in Fig. 1.

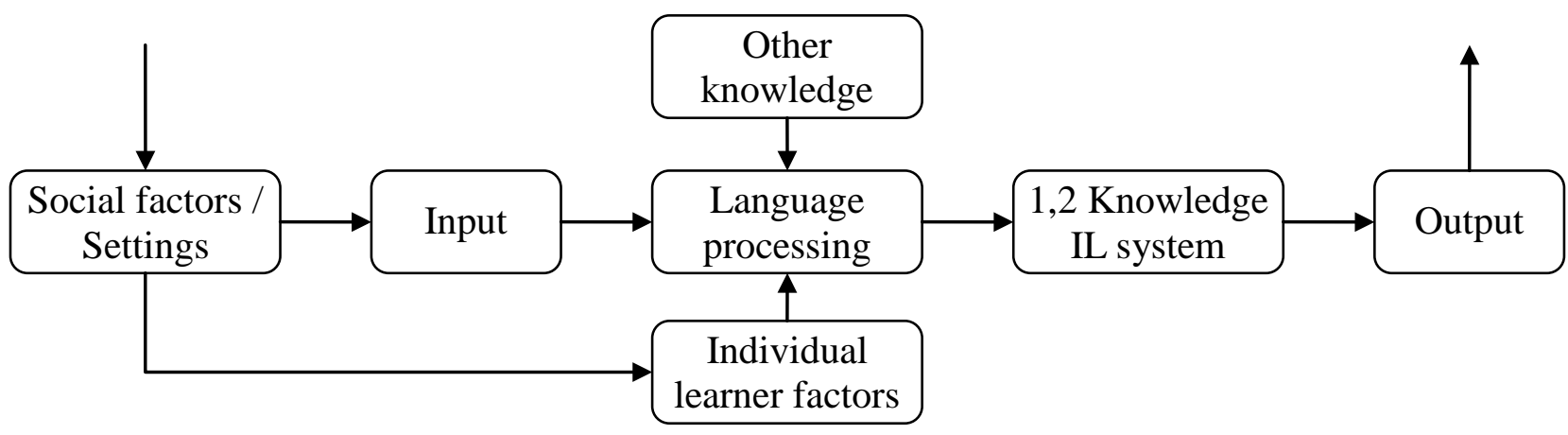

Fig. 1. Principle on second language acquisition theory

Krashen's second language acquisition theory is composed of five hypotheses: namely acquisition-learning hypothesis, monitor hypothesis, natural order hypothesis, input hypothesis and affective-filter hypothesis. The main contents include the following four aspects:

(1) From the perspective of the "acquisition" and "learning" to analyze second language learning, emphasizes the role of understanding significance in language learning. Language structure is built up on the basis of the understanding content, from the side to reflect the importance of language communication; education should meet the needs of learner-centered, teaching should meet the needs of learners as the center, to promote student-centered teaching methods. This view helps to understand the mother tongue and foreign or second language learning differences, enrich the language cognitive theory, and provide a theoretical basis for foreign language teaching and foreign language teaching method.

(2) The input hypothesis is the understanding of the corpus in the first place, that comprehensible input is the premise and the core of the second language acquisition. Comprehensible input is helpful to language ability cultivation; language acquisition is through listening, reading and understanding the corpus information to achieve. For the selection and optimization of language materials provide theoretical support, which has broken the influence of the traditional structural linguistics, and placed the meaning and communication in core position of the language learning.

(3) The significance of the affective-filter hypothesis from the perspective of human nature to explain the factors restricting the acquisition of language learners, and to some extent, reflecting to the importance of the relationship between teachers and students, classroom structure, teaching style, teaching methods, classroom design and teachers' role, and other factors in language acquisition, effective design the above factors, and organize the students' motivation and self-confidence, and reduce anxiety have a great of significance, which will help to improve language skills.

(4) The natural order hypothesis holds that the acquisition of linguistic structure knowledge can be predicted according to the order, regardless of language acquisition or second language or foreign language, the structure of the acquisition grammar knowledge is regular, by more emphasis on grammatical structures that will slow language acquisition, language learning should focus on the understanding what they have learned the material sense and should not be too much emphasis on understanding the grammatical structure.

The main point of second language acquisition theory: language mainly learn through imitation; syntax error occurs when a child, parents usually correct; people with high IQs are good language learners; the best predictor index of success in the second language acquisition is motivation; second language learning begin sooner, learn more likely to be successful; most errors occur second language learners interference from mother tongue; the best way to learn new vocabulary through reading learning; the second language learners master one thousand words and basic grammatical structures can easily talk with native speakers; teachers should first talk about a grammar point, after learners finished, reprocessing the next practice language points; teachers should first speak easy grammar, then telling complex grammar; the second language learner errors should be 
corrected immediately to prevent the formation of bad habits; teachers should use only included students have learned the material structure of language; when the learner allow to freely interact and learn from each other's mistakes; students only learning things taught; The feedback of teachers to the students' errors should be used to repeat what they say in the correct form, rather than explicitly point out the error lies; when using a second language teach other courses, students can simultaneously learn the language and academic content.

\section{Learning Cognitive Theory}

Learning theory can be roughly divided into behavioral theory and cognitive theory two theories. Since the 1950s, the cognitive school turned to the study of cognitive internal cognitive processes, has become the opposition faction of behavioral school. Cognitive psychologists believe that the environment provides only potential irritants, as to whether these stimuli cause and cause what kind of response will have to depend on the internal psychological structure of the learner [4]. Cognitive learning theory include: error analysis theory, input theory, interlanguage theory, constructivist theory, information processing theory, connecting theory. The common feature is the emphasis on learning through insight or awareness of the situation and the formation of cognitive structure to achieve, and advocated the study of internal conditions and internal learning process. The structure of English learning cognitive theory is shown in Fig. 2.

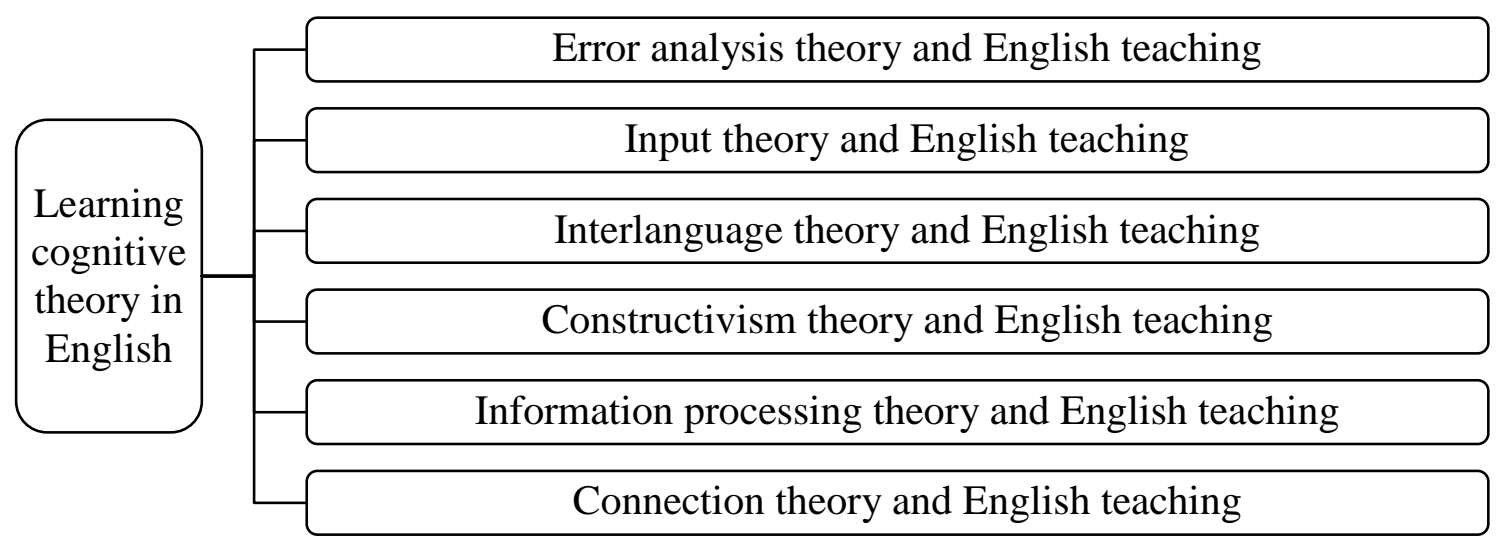

Fig. 2. Structure on learning cognitive theory in English

(1) Error analysis theory. It is a branch of applied linguistics, produced in the late 1960s and the early 1970s. In order to make a systematic analysis of the errors of the learners, we can identify the source of errors, and provide the basis for eliminating these errors in the teaching process. A new theory of analysis language errors "error analysis theory" is produced. English learners make the mistakes in the process of learning by the systematic analysis and research; teachers can obtain useful information, find deficiencies of teaching methods, so as to improve teaching methods, improve teaching effect.

(2) Input theory. Input is a prerequisite for language acquisition, used to trigger internal language acquisition of learners, to explain the relationship between the input and language acquisition from different angles, and stressed the important role of language input. Theoretically recognized in foreign language teaching process should pay full attention to in the different ways the language information input under different circumstances, in the teaching process and in process of a foreign language learning, it should be possible to provide this conditions for the learner; language environment determine the quality of language input, in the process of language input, it should provide learners a more authentic language environment.

(3) Interlanguage theory. Interlanguage, refers to the second language acquisition process, the learner through a certain learning strategies on the basis of a target language input formed on a language, which is different from the first language and their target language, with the progress of learning, the target language to the gradual transition of dynamic language system. "Interlanguage" 
is the independent of language system between native language and target language, is created language system by the second language learners.

(4) Constructivism theory. Constructivism is a theory about knowledge and learning, emphasizing learner's initiative, and considers that learning is learner generated based on the original meaning of knowledge and experience, understand the construction process, and this process is often done in the social and cultural interaction. Constructivism made a profound ideological origins, has quite different from traditional learning theory and teaching ideas, important guiding value for instructional design.

(5) Information processing theory. Human learner is regarded as an information processor, with a computer in a similar manner to operate, when learning occurs, the information entered by the environment, then processed and stored in the memory, the final output in some form. Foreign language learning as a process and language skills in the process become increasingly automated. It focuses on the process of learning how to deal with learner language input, what factors restrict the processing of language input, and how these factors change with time.

(6) Connection theory. Connection theory emphasizes that the complex behavior is a complex reaction, which is establish based on conditioned connection, learning is the process of establishing the connection between the stimulus and the response. Connection theory of learning psychological problems made very valuable insights to guide education and learning practices play an important role. The flaw is that it ignores the deep analysis of the inner psychological process of learning, and it is suitable for explaining the low level learning of animals and human beings.

\section{Intercultural Communication Theory}

Through the integration of these features, including intercultural communication attitudes, intercultural knowledge and intercultural behavioral skills, the system's ICC theory model is established, which includes intercultural communicative ability cognitive, intercultural communication emotion and to participate in intercultural communication competence. In this model, intercultural communication competence is divided into three integrated concept dimensions, namely, intercultural cognitive, intercultural sensitivity, and intercultural proficiency, respectively, representing the above three capabilities, the model structure is shown in Fig. 3.

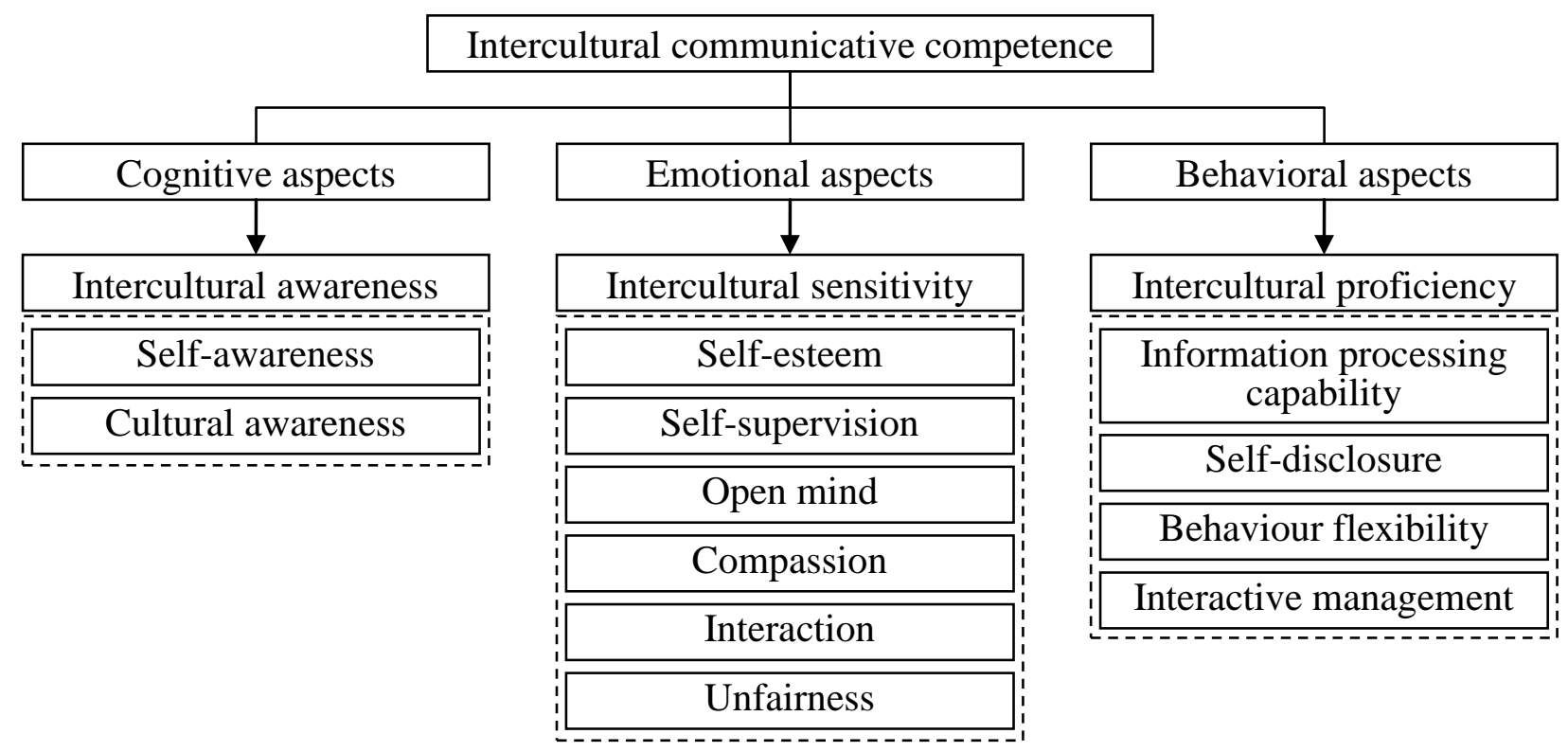

Fig. 3. Theoretical framework on three dimensional model for intercultural communicative competence

The cognitive aspect of intercultural communication competence is namely intercultural awareness, which involves influence people's thinking and cultural practices. Cultural awareness requires people to understand the similarities and differences between cultures and their own culture, 
which is composed of two parts: the self-awareness and cultural awareness; the emotional aspects of intercultural communicative competence that is culturally sensitive, suggesting that the people themselves understand, appreciate and accept different cultural behavior requires. The self-esteem, self-monitoring, an open mind, compassion, mutual influence and unfair; behavior of intercultural communicative competence that is intercultural proficiency, which involves in intercultural communication, so that their behavior has the ability of validity and appropriateness. Focus on how to accomplish the work and how to achieve the goal of intercultural communication through verbal and nonverbal behavior; it is composed of four parts, which are information processing ability, self disclosure, behavioral flexibility and interaction management [5, 6].

Three dimensions are independent of each other, the three combined to make individual to achieve intercultural communication skills. Successful intercultural communication through learning the similarities and the differences between multiple cultures is to improve intercultural communication. Through intercultural sensitivity is to promote the formation of intercultural awareness. The effectiveness and appropriateness of the behavior performance by cognitive and understand the cultural similarities and differences between each other, that is sensitive to adjust. Intercultural awareness and intercultural sensitivity are is a prerequisite for successful intercultural communication. Intercultural sensitivity is the essential factor of intercultural communicative competence and has a significant impact on intercultural awareness and intercultural sensitivity.

\section{Conclusion}

With the advance of globalization, the communication between people from different cultural backgrounds is becoming more and more frequent, and the collision of different regional culture is more and more, this new cultural fusion has been widely concerned by many linguists, has become a hot research topic in pragmatics and applied linguistics. A variety of language skills is a necessary means of communication, some of the language and cultural knowledge, social background knowledge is an indispensable factor in communication. Because there are differences between the various national languages and cultures, people of different countries and regions in intercultural communication process will inevitably produce errors, caused by intercultural communication pragmatic failure, the impact of communication effects. Contents of this paper to solve the core issues of pragmatic failure research in the intercultural communication field, has a very important meaning in-depth study of pragmatic failure in intercultural communication as well as English teaching.

\section{References}

[1] Maria Economidou-Kogetsidis, "Pragmatic failure in non-native speakers' e-mail requests to faculty," Journal of Pragmatics, vol. 43, no. 13, pp. 3193-3215, 2011.

[2] Y. L. Zhu, "Study of cross cultural pragmatic failure from the perspective of adaptation theory," Research on Higher Education in Heilongjiang, vol. 33, no. 9, pp. 174-176, 2014.

[3] Baidu Encyclopedia, "Learning Theory," http://wenku.baidu.com/link?url=dnmPn8EzKQ2FVFTVZ6PrSCKc8E8O1PHK16y2S6fCCUB wsRrTIiQgkmbB977CrNqqr1I184ukLj9zSTbHKavjrjkcMXO84ikDOCDUMhFJyU3, 2015-8-1.

[4]
Baidu
Encyclopedia,
"Intercultural
Communication

Theory," http://doc.mbalib.com/view/fd2fb9eee0b4b38f4a644b3b1bcbee82.html, 2015-8-1.

[5] L. Wu, "Pragmatic failure and preventive strategies in cross cultural communication," Educational theory and Practice, vol. 34, no. 24, pp. 46-47, 2014.

[6] Leonarda Garcia-Jimenez, "The Pragmatic Metamodel of Communication: A cultural approach to interaction," Studies in Communication Sciences, vol. 14, no. 1, pp. 86-93, 2014. 